\title{
Near-Wall Molecular Ordering of Dilute Ionic Liquids
}

\author{
Monchai Jitvisate and James R. T. Seddon*(1) \\ Nanoionics, MESA+ Institute for Nanotechnology, University of Twente, P.O. Box 217, 7500 AE Enschede, The Netherlands
}

Supporting Information

ABSTRACT: The interfacial behavior of ionic liquids promises tunable lubrication as well as playing an integral role in ion diffusion for electron transfer. Diluting the ionic liquids optimizes bulk parameters, such as electric conductivity, and one would expect dilution to disrupt the nearwall molecular ordering. We study this ordering in the ionic liquids $[\mathrm{Emim}]^{+}\left[\mathrm{NTf}_{2}\right]^{-},[\mathrm{Emim}]^{+}[\mathrm{DCA}]^{-}$, and $\left[\mathrm{C}_{4} \mathrm{mpyr}\right]^{+}\left[\mathrm{NTf}_{2}\right]^{-}$, diluted in the solvent dimethyl sulfoxide. We found a structural crossover from well-ordered ionic

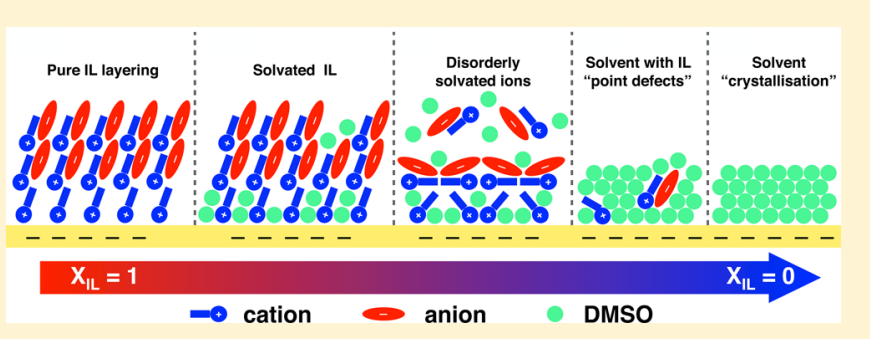
liquids to a well-ordered solvent with increasing dilution, but this occurs nonlinearly, with solvent molecules initially space-filling and solvating and later disrupting the ionic layers. This is of key importance for ionic liquids as optimized tunable nanolubricants.

\begin{abstract}
Tonic liquids (ILs) are organic salts in the liquid phase at 1 room temperature, with the direct result that they have high ionic strength, low volatility, high temperature stability, and inflammability. ${ }^{1,2}$ This has allowed them to find excellent use in energy harvesting and storage technologies, as well as promising switchable lubrication in nanoconfinement. For these applications, one of the most important properties of ILs is their interfacial behavior: It is well accepted that they form discrete molecular layers at solid/liquid interfaces, formed through the disruption of hydrogen-bonded networks within the liquid, for example. ${ }^{3-14}$ The intricate near-wall behavior of the ions then plays a governing role in interlayer slip, as well as in controlling accessibility to an underlying electrode for electron transfer. ${ }^{2,15-17}$
\end{abstract}

Despite the vast literature base on IL ordering in the nearwall region, investigations have so far been almost exclusively related to pure ILs. However, in real applications, it is unlikely that ILs would be used in their pure form. The high viscosity counteracts the high ion concentration, so the overall electrical conductivity is relatively low. ${ }^{18}$ Furthermore, pure ILs remain economically unfeasible. This means that the future will depend more heavily on dilute ILs, which are relatively inexpensive and overcome the practical limitations listed above. To date, only a small number of studies in the literature have focused on the interfacial behavior of dilute ILs, but, even then, most use water or acetonitrile (ACN) as the solvent. ${ }^{19,20}$ These solvents are both highly volatile and limited in electrochemical window $(\mathrm{ECW}),{ }^{21}$ which means that they are not desirable because they remove the main reasons for choosing ILs as electrolytes in the first place. A possible way forward is to use dilute ILs but with a solvent that has a low volatility and large ECW. Dimethyl sulfoxide (DMSO) is one such solvent. It is polar and aprotic and overcomes the limitations of both $\mathrm{ACN}$ and water in terms of both volatility and ECW. ${ }^{21-23}$ Further, it is miscible with a wide range of ILs, hence offering great practicality for applications.
Here, we use force-distance (FD) spectroscopy with an atomic force microscope (AFM) to study the near-wall structure of the ILs 1-ethyl-3-methylimidazolium bis(trifluoromethylsulfonyl)imide $\left([\mathrm{Emim}]^{+}\left[\mathrm{NTf}_{2}\right]^{-}\right)$, 1-ethyl-3methylimidazolium dicyanamide $\left([\text { Emim }]^{+}[\mathrm{DCA}]^{-}\right)$, and 1butyl-1-methylpyrrolidinium bis(trifluoromethylsulfonyl)imide $\left(\left[\mathrm{C}_{4} \mathrm{mpyr}\right]^{+}\left[\mathrm{NTf}_{2}\right]^{-}\right)$, solvated by DMSO, on a mica wall. Mica was chosen as the substrate because it is atomically flat with a well-defined charge per unit area. It has an octahedral crystal structure, with the unit cell parameters $a=0.519 \mathrm{~nm}, b=0.901$ $\mathrm{nm}$, and $c=2.00 \mathrm{~nm}$. In its fully dissociated condition, it contains an average area per negative charge site of $0.47 \mathrm{~nm}^{2}$ (approximate surface charge of $2 \mathrm{e} / \mathrm{nm}^{2}$ ). ${ }^{24}$ However, the surface charge of mica in ionic liquids is still not truly known. According to recent electrical double layer potential measurements using surface force apparatus, the calculated surface charges of mica at $22{ }^{\circ} \mathrm{C}$ in the ionic liquids $\left[\mathrm{C}_{2} \mathrm{mim}\right]^{+}\left[\mathrm{NTf}_{2}\right]^{-}$ and $\left[\mathrm{C}_{3} \mathrm{mim}\right]^{+}\left[\mathrm{NTf}_{2}\right]^{-}$are $0.28 \mathrm{C} / \mathrm{m}^{2}\left(\sim 1.76 \mathrm{e} / \mathrm{nm}^{2}\right)$ and 0.19 $\mathrm{C} / \mathrm{m}^{2}\left(\sim 1.16 \mathrm{e} / \mathrm{nm}^{2}\right)$, respectively. ${ }^{25}$ From these values, it is clear that the mica surface in pure ionic liquids is close to fully dissociated. The three ILs were chosen to allow conclusions to be drawn on the effects of both cations and anions. We found nonlinear near-wall structural changes upon dilution that appear to correlate with solvent molecules initially space-filling and solvating and later disrupting the ionic layers.

We begin by presenting the FD spectroscopy data for the $100 \%, 50 \%$, and $0 \%$ mixtures of $[\text { Emim }]^{+}\left[\mathrm{NTf}_{2}\right]^{-}$with DMSO in Figure 1 (see Figure S1 for all dilutions studied for each of the ILs). The $x$ axes represent the separation between the substrate and AFM tip, with the substrate located at $0 \mathrm{~nm}$. The $y$ axes represent the tip-sample interaction due to the squeeze film of IL between tip and wall, that is, the force (sometimes

Received: May 19, 2017

Revised: August 8, 2017

Published: August 8, 2017 

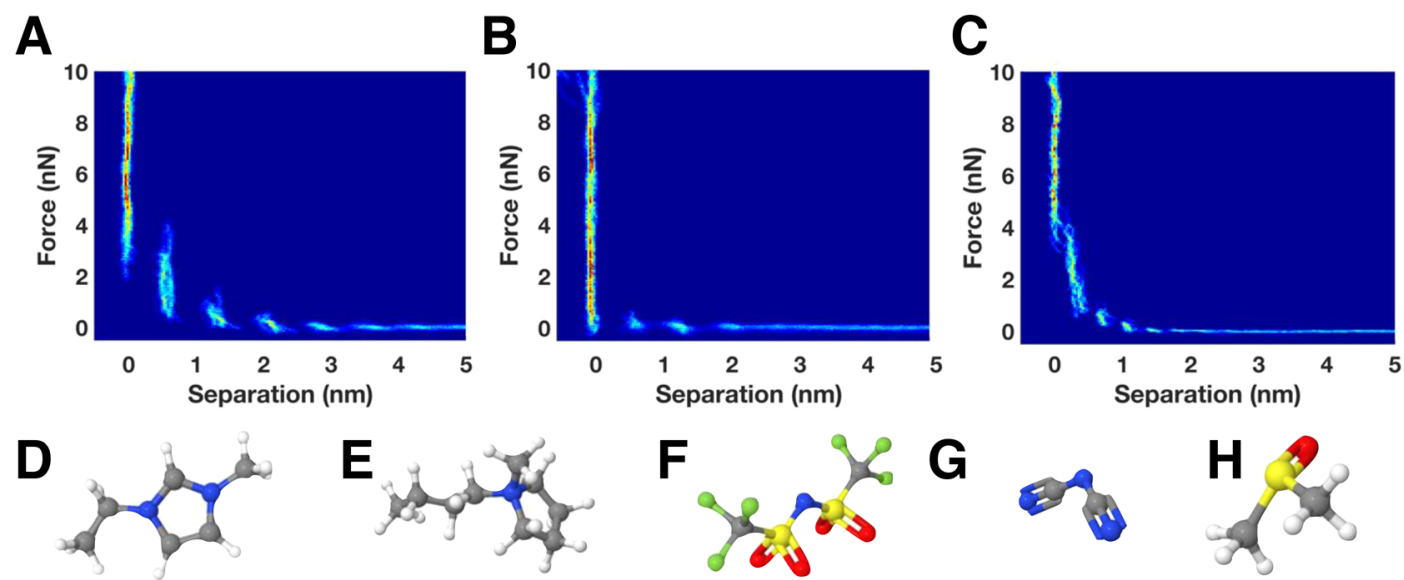

Figure 1. (A-C) Force-distance spectroscopy of $[\mathrm{Emim}]^{+}\left[\mathrm{NTf}_{2}\right]^{-} / \mathrm{DMSO}$ mixtures, at IL concentrations of (A) $100 \%$, (B) $50 \%$, and (C) $0 \%$. Discrete molecular layers of the liquids form on the mica wall and extend several nanometers into the bulk. (D-H) The molecules used in this study are (D) $[\mathrm{Emim}]^{+},(\mathrm{E})\left[\mathrm{C}_{4} \mathrm{mpyr}\right]^{+},(\mathrm{F})\left[\mathrm{NTf}_{2}\right]^{-},(\mathrm{G})[\mathrm{DCA}]^{-}$, and (H) DMSO.
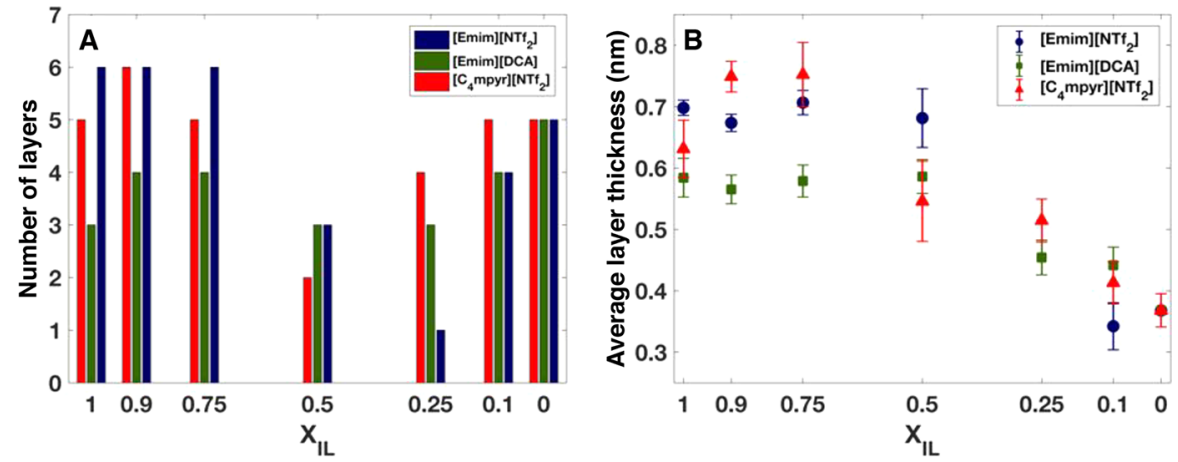

Figure 2. (A) Number of layers and (B) average layer thickness as a function of IL mole fraction. The average layer thickness is calculated from the first three layers. The mixtures have fewer of layers at medium dilutions because of structural switching from high to low IL concentration.

referred to as the structural force) exerted by the liquid on the AFM tip. As the tip approaches the wall, a series of discrete steps occurs, corresponding to ordered layers of liquid molecules on the mica surface. The fact that this near-wall molecular ordering can be seen is because it exhibits quite a large degree of rigidity: To push through each layer, the AFM cantilever must bend to store sufficient (elastic) energy to rupture the layer; then the liquid molecules "pop" out, and the tip jumps to the next layer.

Liquid layering occurs in both ILs and DMSO because the molecules are templated by the underlying atomically smooth crystal structure of mica. ${ }^{26} \mathrm{We}$ plot the number of layers observed for each of our IL dilutions in Figure 2A. The first data we describe are for the pure systems, namely, the pure ILs at a concentration percentage of $X_{\mathrm{IL}}=1$ and the pure solvent at $X_{\mathrm{IL}}=0$. In all cases, the pure liquids form several well-ordered layers. However, it is surprising that the different ILs exhibit different numbers of layers. We naively expected the first layer to be predominantly filled with cations (due to the dissociation of $\mathrm{K}^{+}$ions from the mica surface) and to template subsequent layers. This would intuitively lead to the two ILs with similar cations $\left([\mathrm{Emim}]^{+}\left[\mathrm{NTf}_{2}\right]^{-}\right.$and $\left.[\mathrm{Emim}]^{+}[\mathrm{DCA}]^{-}\right)$exhibiting similar near-wall structures. In reality, however, we found that $[\text { Emim }]^{+}\left[\mathrm{NTf}_{2}\right]^{-}$forms six layers whereas $[\text {Emim }]^{+}[\mathrm{DCA}]^{-}$ only forms three. This is a strong indication that the co-ion plays a key role in the layer formation, even when charge screening is required, hinting that templating is more important than charge screening as a driving force for the molecular ordering. The third IL $\left(\left[\mathrm{C}_{4} \mathrm{mpyr}\right]^{+}\left[\mathrm{NTf}_{2}\right]^{-}\right)$sits between the other two in terms of the number of layers it creates in its pure form, exhibiting five distinct layers on mica. It could be that the ratio of cation size to anion size governs the number of layers. This is certainly true for our three ILs, with the more equally sized systems exhibiting more layers than the less equally sized systems, but the complex geometries of the different molecules, as well as the position of the valency along the molecule, must also be taken into account for a full description of this observation.

As we began to dilute the ILs, we found the surprising result that the number of ordered liquid layers increased. By comparing the $X_{\mathrm{IL}}=1$ data to the $X_{\mathrm{IL}}=0.9$ data, one can immediately see that the number of layers of $[\mathrm{Emim}]^{+}[\mathrm{DCA}]^{-}$ increases from three to four, whereas the number of layers of $\left[\mathrm{C}_{4} \mathrm{mpyr}\right]^{+}\left[\mathrm{NTf}_{2}\right]^{-}$increases from five to six. This observation was unforeseen: We expected the addition of solvent to disrupt the near-wall molecular ordering of the IL, that is, to reduce the layering effect, not enhance it. We imagine that this is due to solvation of the cations in the first layer by DMSO molecules. Anions would not sit preferentially in the first layer, which should be cation-rich for charge-screening purposes, ${ }^{11,12,27}$ but also the cations would not be immediately adjacent to each other either because of electrostatic repulsion.

Also, working from the pure DMSO case, where one can see five distinct molecular layers form on the mica, adding a small amount of IL disrupts these layers slightly, and the number of layers drops from five to four for the two $[\mathrm{Emim}]^{+}$ILs. It is 

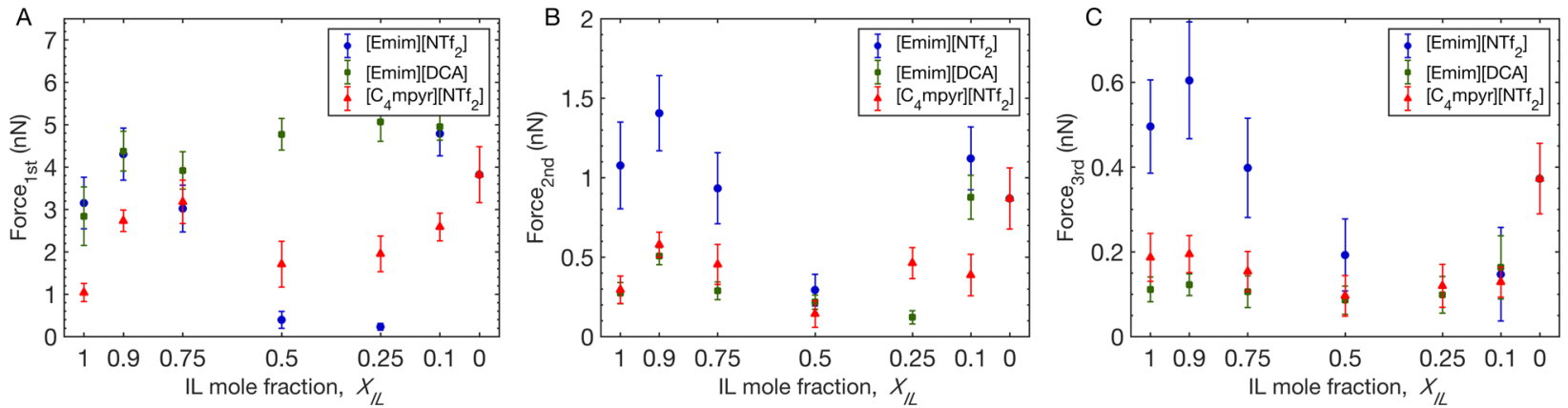

Figure 3. Average rupture force required for pushing through (A) the first layer (innermost), (B) the second layer, and (C) the third layer, at corresponding mole fraction. Each data point was averaged over 20 force-distance curves at the highest force of each layer.

clear that layer enhancement should not occur during the dilution of the pure DMSO: It has only one molecule type, and it can pack very well. Any disruption of this ordering should reduce the overall near-wall preferential ordering. Why do the $[\text { Emim }]^{+}$ILs show a larger effect than $\left[\mathrm{C}_{4} \mathrm{mpyr}\right]^{+}$? We believe that the cation charge location is more accessible to the electronegative oxygen of the DMSO on the $[\mathrm{Emim}]^{+}$ion than on the $\left[\mathrm{C}_{4} \mathrm{mpyr}\right]^{+}$ion, allowing the imidazolium ion to penetrate deeper into the layered structure and thus provide more disruption. ${ }^{28,29}$

In addition to the number of layers, the average layer thickness also provides information on the local packing; see Figure 2B. For all pure ILs, the first few layers are slightly thinner than the bulk ion-pair dimensions (see Table S1), but it is also clear that the specific orientation of the cation in the first layer changes depending on the type of anion. As we diluted the ILs, the two imidazolium liquids retained their "pure" layer thickness until a mole fraction of $\sim 0.5$. Below this mole fraction, the layer thickness drops linearly until the pure DMSO is reached at $X_{\mathrm{IL}}=0$. The near-wall structure of the pyrolidinium liquid actually swells during initial dilution, but then it also drops linearly in the weak dilution limit. This linear drop-off is different from the case of the solvent propylene carbonate (PC). ${ }^{30}$ Mixtures of $\left[\mathrm{C}_{4} \mathrm{mpyr}\right]^{+}\left[\mathrm{NTf}_{2}\right]^{-}$and PC on mica exhibit a steplike switch in near-wall geometry with concentration, which indicates that the dependence of local ordering on dilution is solvent-specific.

We now understand that DMSO preferentially solvates cations over anions and that this leads to an enhancement of molecular ordering of ILs on mica for highly concentrated solutions, crossing over to full solvent ordering at weak dilutions. This would be confirmed by an increase in the force required to rupture the near-wall ordered layers as solvent is added. The rupture forces of the first three layers are plotted as a function of IL mole fraction in Figure 3. Among these layers, we expect the first layer immediately adjacent to the mica to be heavily influenced by the surface charge (Figure 3A), that is, electrostatic interaction might be a significant contributory factor for the layer strength of the first layer. In this respect, considering the pure ILs $\left(X_{\mathrm{IL}}=1\right)$, it is perhaps unsurprising that the two $[\mathrm{Emim}]^{+}$cations exhibit the same rupture force, within the error, indicating that the anion is less important in the packing structure in the first layer. The third pure IL, with the pyrolidinium cation, exhibits a smaller rupture force for the first layer than the two imidazolium ILs, and we interpret this effect as resulting from the bulkiness of the ion disrupting packing with its neighbors in the plane.
Now, we turn to the dilute ILs. The common behavior for the first layer of all three ILs is that the rupture force (i) increases when a small amount of DMSO is added, (ii) decreases at medium concentrations, and (iii) then increases again in the very dilute regime. All of these observations agree with our discussion earlier in the article regarding the solvent sitting in voids between adjacent ions at the wall. For example, the initial increase in rupture force upon a small addition of solvent is fully supported if the solvent molecules do indeed sit in the voids, thus increasing the layer packing through spacefilling. At mixtures closer to 50:50 composition, the local packing strength is lost as the liquid become more anisotropic, whereas at weaker dilutions, the strong DMSO packing leads to an increase in strength once more.

The rupture force for the second and third layers exhibits the same overall trend as that for the first layer, with an initial increase in force from a small addition of solvent, then a decrease at intermediate mixture compositions, and then an increase again at almost pure DMSO. The same argument for this behavior in the first layer also holds for these two subsequent layers, but now, $[\mathrm{Emim}]^{+}\left[\mathrm{NTf}_{2}\right]^{-}$is overall stronger than both of the other two ILs. This indicates that the packing beyond the first layer is governed primarily by neither the cation (where we would imagine similarity between $[\text { Emim }]^{+}\left[\mathrm{NTf}_{2}\right]^{-}$and $[\mathrm{Emim}]^{+}[\mathrm{DCA}]^{-}$) nor the anion (where we would imagine similarity between $[\mathrm{Emim}]^{+}\left[\mathrm{NTf}_{2}\right]^{-}$and $\left.\left.\left[\mathrm{C}_{4} \mathrm{mpyr}\right]^{+} \mathrm{NTf}_{2}\right]^{-}\right)$. Instead, we believe that ion pairs are the main constituents that give these layers their strength, with or without solvent, and any additional interactions between the dense electron cloud on the oxygen of the DMSO and the $[\text { Emim }]^{+}$cation is now only secondary.

In summary, we measured the layering structure of the ILs $[\text { Emim }]^{+}\left[\mathrm{NTf}_{2}\right]^{-},[\text {Emim }]^{+}[\mathrm{DCA}]^{-}$, and $\left[\mathrm{C}_{4} \mathrm{mpyr}^{+}\left[\mathrm{NTf}_{2}\right]^{-}\right.$ on mica as a function of dilution with DMSO over the full range of concentrations using FD spectroscopy. We found that the number of ordered liquid layers, layer thickness, and rupture force vary with the mole fraction of IL in DMSO. A small amount of DMSO can increase the strength of the layers. Adding more DMSO leads to a structural crossover from a wellordered IL structure to a well-ordered solvent structure, with the second chemical species acting as point defects disrupting this structure at low concentrations, but this crossover is not switch-like as in the case of the solvent PC. One possible interpretation of our results is that the DMSO solvates the cations and fills the free volume between the IL molecules, with these stronger layers then able to template liquid further into the bulk. This speculative interpretation would explain the observations presented here, but it is clear that extensive 
(molecular dynamics) computation is required for a full understanding.

We believe that our observations are highly relevant for controllable lubrication in nanoconfinement (e.g., nanofluidics, nanoslits, nanopores) where the interplay of adjacent molecular layers can either enhance or restrict local flow properties. We also see relevance in ionic liquid energy technologies where we expect solvation to prove useful for optimizing electrical conductance, for example, but where "crystallization" of solvent must be avoided.

\section{EXPERIMENTAL SECTION}

We used mica (Bruker) as the substrate, which we freshly cleaved immediately prior to use. The ILs were $[\text { Emim }]^{+}\left[\mathrm{NTf}_{2}\right]^{-},[\text {Emim }]^{+}[\mathrm{DCA}]^{-}$, and $\left[\mathrm{C}_{4} \mathrm{mpyr}\right]^{+}\left[\mathrm{NTf}_{2}\right]^{-}$ (>99\%, Merck Millipore), used as received from the manufacturer. These ILs were mixed with anhydrous dimethyl sulfoxide (DMSO) (>99.9\%, Sigma-Aldrich) to form mixtures with IL mole fractions of $1,0.9,0.75,0.5,0.25$, and 0.1 These mole fractions correspond to number density ratios of IL molecules to total molecules in the mixture. All chemicals were kept and prepared in an MBraun LABmaster glovebox, filled with purified nitrogen, and we performed all experiments at 25 ${ }^{\circ} \mathrm{C}$.

We measured the near-wall ion ordering with force-distance (FD) spectroscopy using an Asylum Cypher AFM (Santa Barbara, CA). For each IL dilution, we deposited a droplet of $\sim 80 \mu \mathrm{L}$ on the mica and immediately started measuring. The AFM was placed on a vibration-free bench in the laboratory environment, and each measurement typically took less than 1 h.

The cantilevers were Au-coated $\mathrm{Si}_{3} \mathrm{~N}_{4}$ (ORC8, Bruker). The spring constants, $k_{\mathrm{s}}$, were measured to be between 0.4 and 0.7 $\mathrm{N} / \mathrm{m}$, and the tip radius of curvature, $R$, was quoted to be 15 $\mathrm{nm}$ by the manufacturer. To obtain the force curves, the AFM tip was moved toward the surface using a piezo with the speed of $5 \mathrm{~nm} / \mathrm{s}$ until a predefined trigger point was reached. The voltage of the approach piezo versus deflection of the cantilever measured using a photodiode were converted into tip-wall separation versus force in the usual way. Twenty force curves were collected for each liquid mole fraction in our experiment, and these are plotted as histograms in this article. (As a note, when we discuss the number of layers, this is clearly the number of layers within our resolution, which is approximatley $100 \mathrm{pN}$. We imagine that other layers might exist below this measurement resolution.)

\section{ASSOCIATED CONTENT}

\section{S Supporting Information}

The Supporting Information is available free of charge on the ACS Publications website at DOI: 10.1021/acs.jpcc.7b04843.

FD data for all dilutions of all ILs used in this study. Tabulated data of ion sizes (PDF)

\section{AUTHOR INFORMATION}

\section{Corresponding Author}

*E-mail: j.r.t.seddon@utwente.nl.

ORCID

James R. T. Seddon: 0000-0002-6069-3879

Notes

The authors declare no competing financial interest.

\section{ACKNOWLEDGMENTS}

The authors thank Jens Smiatek for useful discussions on the role of DMSO in solvating ionic liquids. M.J. acknowledges The Development and Promotion of Science and Technology Talents project (DPST) for financial support.

\section{REFERENCES}

(1) Pott, T.; Méléard, P. New Insight into the Nanostructure of Ionic Liquids: A Small Angle X-Ray Scattering (SAXS) Study on Liquid trialkyl-methyl-ammonium bis (trifluoromethanesulfonyl) amides and their Mixtures. Phys. Chem. Chem. Phys. 2009, 11, 5469-5475.

(2) Fedorov, M. V.; Kornyshev, A. A. Ionic Liquids at Electrified Interfaces. Chem. Rev. 2014, 114, 2978-3036.

(3) Morita, T.; Ushio, M.; Kanoh, K.; Tanaka, E.; Nishikawa, K. Small-Angle X-ray Scattering Measurements of Ionic Liquids Pressurized with Carbon Dioxide Using Titanium Sample Holder: 1Butyl-3-methylimidazolium Bis (trifluoromethylsulfonyl) Amide Mixtures up to $22 \mathrm{MPa}$. Jpn. J. Appl. Phys. 2012, 51, 076703.

(4) Uysal, A.; Zhou, H.; Feng, G.; Lee, S. S.; Li, S.; Fenter, P.; Cummings, P. T.; Fulvio, P. F.; Dai, S.; McDonough, J. K.; Gogotsi, Y. Structural Origins of Potential Dependent Hysteresis at the Electrified Graphene/Ionic Liquid Interface. J. Phys. Chem. C 2014, 118, 569574.

(5) Mezger, M.; Ocko, B. M.; Reichert, H.; Deutsch, M. Surface Layering and Melting in an Ionic Liquid Studied by Resonant Soft XRay Reflectivity. Proc. Natl. Acad. Sci. U. S. A. 2013, 110, 3733-3737.

(6) Perkin, S.; Crowhurst, L.; Niedermeyer, H.; Welton, T.; Smith, A. M.; Gosvami, N. N. Self-Assembly in the Electrical Double Layer of Ionic Liquids. Chem. Commun. 2011, 47, 6572-6574.

(7) Perkin, S. Ionic Liquids in Confined Geometries. Phys. Chem. Chem. Phys. 2012, 14, 5052-5062.

(8) Smith, A. M.; Lovelock, K. R.; Gosvami, N. N.; Welton, T.; Perkin, S. Quantized Friction Across Ionic Liquid Thin Films. Phys. Chem. Chem. Phys. 2013, 15, 15317-15320.

(9) Atkin, R.; El Abedin, S. Z.; Hayes, R.; Gasparotto, L. H.; Borisenko, N.; Endres, F. AFM and STM Studies on the Surface Interaction of [BMP] TFSA and [EMIm] TFSA Ionic Liquids with $\mathrm{Au}$ (111). J. Phys. Chem. C 2009, 113, 13266-13272.

(10) Hayes, R.; Warr, G. G.; Atkin, R. At the Interface: Solvation and Designing Ionic Liquids. Phys. Chem. Chem. Phys. 2010, 12, 17091723.

(11) Hayes, R.; Borisenko, N.; Tam, M. K.; Howlett, P. C.; Endres, F.; Atkin, R. Double Layer Structure of Ionic Liquids at the Au (111) Electrode Interface: An Atomic Force Microscopy Investigation. J. Phys. Chem. C 2011, 115, 6855-6863.

(12) Black, J. M.; Walters, D.; Labuda, A.; Feng, G.; Hillesheim, P. C.; Dai, S.; Cummings, P. T.; Kalinin, S. V.; Proksch, R.; Balke, N. Bias-Dependent Molecular-Level Structure of Electrical Double Layer in Ionic Liquid on Graphite. Nano Lett. 2013, 13, 5954-5960.

(13) Seddon, J. R. Conservative and Dissipative Interactions of Ionic Liquids in Nanoconfinement. J. Phys. Chem. C 2014, 118, 2219722201.

(14) Hayes, R.; Warr, G. G.; Atkin, R. Structure and Nanostructure in Ionic Liquids. Chem. Rev. 2015, 115, 6357-6426.

(15) Kornyshev, A. A. Double-Layer in Ionic Liquids: Paradigm Change? J. Phys. Chem. B 2007, 111, 5545-5557.

(16) Smith, A. M.; Lovelock, K. R.; Perkin, S. Monolayer and Bilayer Structures in Ionic Liquids and their Mixtures Confined to NanoFilms. Faraday Discuss. 2014, 167, 279-292.

(17) Jitvisate, M.; Seddon, J. R. Local Structure and Flow Properties of Ionic Liquids on Charged and Inert Substrates. J. Phys. Chem. C 2016, 120, 4860-4865.

(18) Chaban, V. V.; Voroshylova, I. V.; Kalugin, O. N.; Prezhdo, O. V. Acetonitrile Boosts Conductivity of Imidazolium Ionic Liquids. J. Phys. Chem. B 2012, 116, 7719-7727.

(19) Wu, X.; Liu, Z.; Huang, S.; Wang, W. Molecular Dynamics Simulation of Room-Temperature Ionic Liquid Mixture of [bmim][BF 
4] and Acetonitrile by a Refined Force Field. Phys. Chem. Chem. Phys. 2005, 7, 2771-2779.

(20) Wang, Z.; Li, H.; Atkin, R.; Priest, C. Influence of Water on the Interfacial Nanostructure and Wetting of [Rmim][NTf2] Ionic Liquids at Mica Surfaces. Langmuir 2016, 32, 8818-8825.

(21) Marcus, Y. The Properties of Solvents; Wiley: Chichester, U.K., 1998; Vol. 16.

(22) Laoire, C. O.; Mukerjee, S.; Abraham, K.; Plichta, E. J.; Hendrickson, M. A. Influence of Nonaqueous Solvents on the Electrochemistry of Oxygen in the Rechargeable Lithium-Air Battery. J. Phys. Chem. C 2010, 114, 9178-9186.

(23) Krishnamoorthy, A. N.; Zeman, J.; Holm, C.; Smiatek, J. Preferential Solvation and Ion Association Properties in Aqueous Dimethyl Sulfoxide Solutions. Phys. Chem. Chem. Phys. 2016, 18, 31312-31322.

(24) Christenson, H. K.; Thomson, N. H. The Nature of the AirCleaved Mica Surface. Surf. Sci. Rep. 2016, 71, 367-390.

(25) Gebbie, M. A.; Valtiner, M.; Banquy, X.; Fox, E. T.; Henderson, W. A.; Israelachvili, J. N. Ionic Liquids Behave as Dilute Electrolyte Solutions. Proc. Natl. Acad. Sci. U. S. A. 2013, 110, 9674-9679.

(26) Israelachvili, J. N. Intermolecular and Surface Forces, 3rd ed.; Academic Press: San Diego, CA, 2011.

(27) Lynden-Bell, R. M.; Frolov, A.; Fedorov, M. V. Electrode Screening by Ionic Liquids. Phys. Chem. Chem. Phys. 2012, 14, 26932701.

(28) Zhao, H. Protein Stabilization and Enzyme Activation in Ionic Liquids: Specific Ion Effects. J. Chem. Technol. Biotechnol. 2016, 91, 25-50.

(29) Schröder, C. Proteins in Ionic Liquids: Current Status of Experiments and Simulations. Top. Curr. Chem. 2017, 375, 25.

(30) Smith, A. M.; Lee, A. A.; Perkin, S. Switching the Structural Force in Ionic Liquid-Solvent Mixtures by Varying Composition. Phys. Rev. Lett. 2017, 118, 096002. 